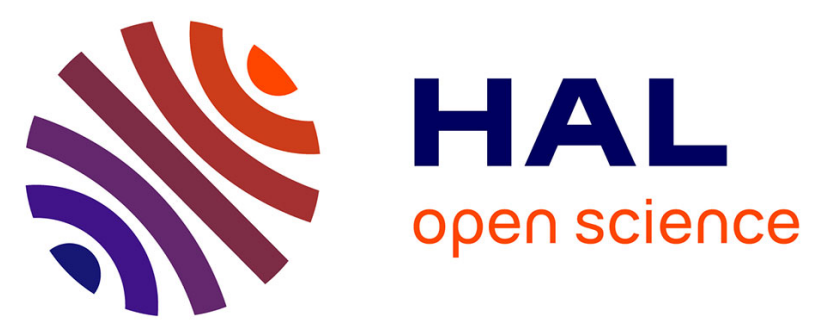

\title{
Energy and Resource Allocations for Battery Aging-Aware Green Cellular Networks
}

\author{
Ali El Amine, Hussein Al Haj Hassan, Loutfi Nuaymi
}

\section{To cite this version:}

Ali El Amine, Hussein Al Haj Hassan, Loutfi Nuaymi. Energy and Resource Allocations for Battery Aging-Aware Green Cellular Networks. 2018 IEEE Global Communications Conference: Green Communications Systems and Networks (Globecom2018 GCSN), Dec 2018, Abu Dhabi, United Arab Emirates. 10.1109/GLOCOM.2018.8647197 . hal-01988211

\section{HAL Id: hal-01988211 https://imt-atlantique.hal.science/hal-01988211}

Submitted on 21 Jan 2019

HAL is a multi-disciplinary open access archive for the deposit and dissemination of scientific research documents, whether they are published or not. The documents may come from teaching and research institutions in France or abroad, or from public or private research centers.
L'archive ouverte pluridisciplinaire HAL, est destinée au dépôt et à la diffusion de documents scientifiques de niveau recherche, publiés ou non, émanant des établissements d'enseignement et de recherche français ou étrangers, des laboratoires publics ou privés. 


\title{
Energy and Resource Allocations for Battery Aging-Aware Green Cellular Networks
}

\author{
Ali El-Amine *, Hussein Al Haj Hassan ${ }^{\dagger}$, Loutfi Nuaymi * \\ ${ }^{\star}$ Departement Système Réseaux, Cybersécurité et Droit du numérique (SRCD). IMT-Atlantique. Rennes, France \\ ${ }^{\dagger}$ Department of Computer and Communications Engineering. Faculty of Engineering. American University of \\ Science and Technology. Beirut, Lebanon \\ Email: *firstname.lastname@imt-atlantique.fr, †hhajhassan@aust.edu.lb
}

\begin{abstract}
In this paper, we study the grid energy consumption of a hybrid energy powered base station system equipped with a renewable source and a battery. We focus on the energy storage element that is prone to irreversible aging mechanisms, thus requiring precise management that takes into account both energy cost and constraints preventing fast battery degradation. We propose a Battery Aging and Price-Aware (BAPA) algorithm that brings down the grid energy consumption of the operator while including battery degradation constraints. We decompose the problem into three subproblems: Resource allocation problem, grid energy purchase problem and power allocation problem. We show that our algorithm performs very close to optimality (up to $99 \%$ ) and outperforms a benchmark algorithm.
\end{abstract}

\section{INTRODUCTION}

In order to meet the challenges of high energy demand and $\mathrm{CO}_{2}$ emissions, green cellular networks with improved energy efficiency have become a key factor in future $5 \mathrm{G}$ cellular networks. According to the power consumption breakdown in [1], the major part of energy demand comes from the base station (BS), consuming more than $50 \%$ of the power of a cellular network. Therefore, reducing the grid power at this level is important for economic and ecological concerns. For instance, it is estimated that Information and Communication Technology (ICT) consumes around $4.7 \%$ of the world's electrical energy, releasing into the atmosphere about $1.7 \%$ of the global $\mathrm{CO}_{2}$ emissions [2]. Among the different energy saving solutions proposed for wireless communication, providing energy harvesting capabilities to cellular BSs from renewable energy (RE) sources is another effective approach to increase the energy savings and reduce the carbon footprint.

A lot of studies have investigated the use of RE in wireless cellular networks as an economic and environmental friendly technique that can harvest clean and cheap energy from ambient surrounding (see [3] and references therein). Since this energy source is intermittent, a battery is used as an energy storage to address this limitation and add flexibility in the energy utilization. However, most of these studies focus on brining down the grid energy consumption without taking into account the health degradation of the battery that represents a significant cost. For example, we showed in [4] that managing the use of RE according to the price of electricity and the battery state of charge (SoC) in a hybrid power BS system can significantly bring down the operational cost for the operator. We further extended this work in [5] to cover cellular networks equipped with RE and batteries. By adjusting the network configuration, we were able to increase the utilization of RE, resulting in further reduction in the grid energy consumption. Enabling energy cooperation between BSs equipped with RE to maximize the network energy efficiency is addressed in [6]. Under the smart grid infrastructure, the authors showed that by exchanging the harvested energy between BSs, more improvement in the network energy savings is observed.

However, a typical battery requires expensive investment cost and is prone to irreversible degradations. Some of these degradations are subject to energy exchanges with the battery, and are known as cycle aging. Others occur when the battery is at rest, and they are known as calendar aging. Among the few studies that consider the aging of batteries while studying cellular networks equipped with RE is [7]. This study demonstrates that by taking into account the necessary requirements that prevent the battery from the above mentioned degradations, its state of health can extend up to $50 \%$ per year, resulting in an increase in the opex cost savings. However, this work considers only offline solution. Hence, it is limited to non-causal information of the RE generation.

In this paper, we study the grid energy price reduction of a BS powered by a hybrid energy source (RE and smart grid) in a variable electricity price environment. We decompose the problem into three sub-problems: resource sharing problem, grid energy purchase problem and power allocation problem. In contrast to [7], we propose an online algorithm that does not require the knowledge of future RE generation. Then, we demonstrate the efficiency of our solution by comparing it with the optimal offline approach. We show that our algorithm can reach up to $99 \%$ optimality, and outperforms an online benchmark algorithm.

\section{System Operation MOdEL}

\section{A. System Architecture}

As illustrated in Fig. 1, we consider an architecture composed of: 
- $\quad$ A BS covering an area of radius $\mathrm{R}$ and serving $K$ mobile users. Let $\mathcal{U}=\{1, \ldots, K\}$ be the set of served users. The users request a minimum data rate of $R^{r e q}$.

- A RE source (Photo-Voltaic (PV) system) that harvests solar energy to produce electricity.

- A battery to store energy either from the PV system or the power grid (i.e., smart grid). This storage unit allows flexibility in the energy utilization.

- The smart grid as the second source in providing energy to the system. It can directly power the BS or get stored in the battery for future use (i.e., when the price of electricity is low).

- A local Energy Management Unit (EMU) responsible for managing the flow of energy, and allocating power (grid and RE) to efficiently maximize the utilization of RE, and thus minimize the grid energy cost.

The amount of RE generated during time $t$, denoted by $E(t)$, is stored in the battery $\left(E^{\mathrm{b}}(t)\right)$ and is also available for use directly $\left(E^{\mathrm{BS}}(t)\right)$. Similarly, the amount of grid power purchased from the smart grid is used to power the $\mathrm{BS}\left(p^{\mathrm{g}, \mathrm{BS}}(t)\right)$ and, if necessary, to store in the battery $\left(p^{\mathrm{g}, \mathrm{b}}(t)\right)$ for future use. This results in the following equalities:

$$
\begin{gathered}
E(t)=E^{\mathrm{BS}}(t)+E^{\mathrm{b}}(t)+w(t) \\
p^{\mathrm{g}}(t)=p^{\mathrm{g}, \mathrm{b}}(t)+p^{\mathrm{g}, \mathrm{BS}}(t)
\end{gathered}
$$

where $w(t)$ is the amount of waste in RE.

The local EMU manages the allocation of RE, whether to be used or stored in the battery. It also decides when and how much to store grid energy.

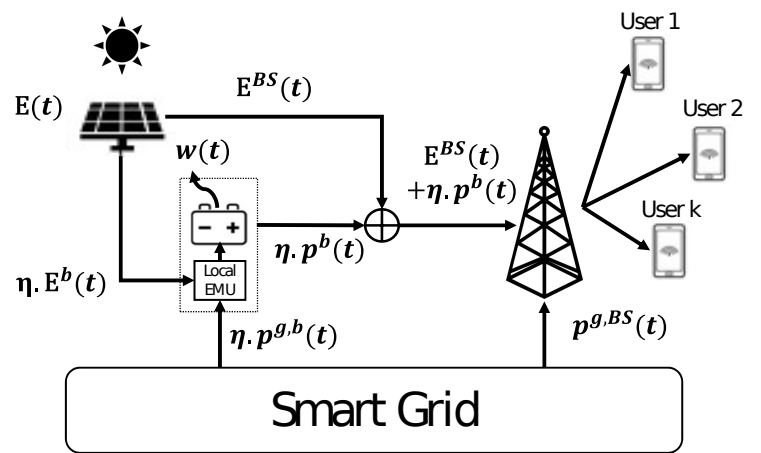

Fig. 1: System architecture. The BS serves $K$ users and is powered by a hybrid energy source.

\section{B. Time Scales for Network Operations}

Due to the fast variations of the traffic load distribution and the downlink wireless channels compared to the slow variations of the grid energy prices and the amount of harvested RE, we consider two time scales in our model. One is longer than the other. As shown in
Fig. 2, each large-time scale period is decomposed into $L$ time slots. During these time slots, only the channel gains and the position of the users vary. We define the large-time scale periods long enough so that the traffic load, the RE arriving rate and the price of electricity are unchanged during each of these time periods.

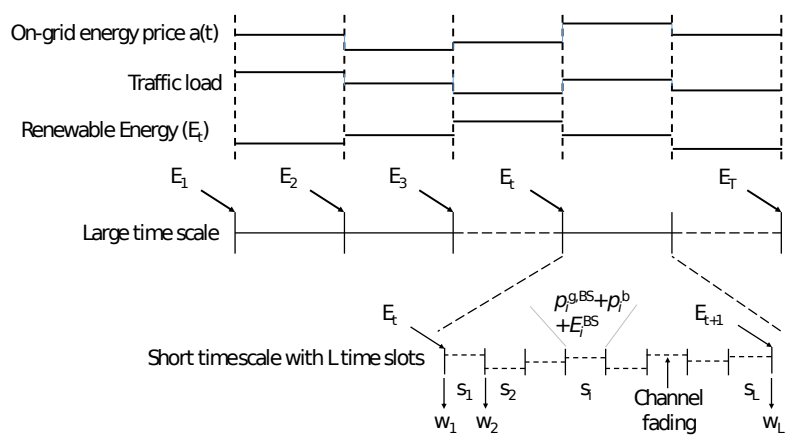

Fig. 2: System time scales. The price of electricity, the traffic load and the RE arrivals vary slowly compared to the channel fading and users distribution.

\section{Downlink Transmission Model}

We measure the downlink transmission quality between the serving BS and a user $m$, based on the SNR as follows:

$$
\operatorname{SNR}(m)=\frac{P^{\mathrm{Tx}} \times h(m)}{\sigma^{2}}
$$

where $P^{\mathrm{Tx}}$ is the transmitted power of the $\mathrm{BS}, h(m)$ is the channel gain from the BS to user $m$, which accounts for the path loss and shadowing effect, and $\sigma^{2}$ is the additive white Gaussian noise power density.

We express the rate offered to user $m$ and served by the BS using Shannon-Hartley theorem as follows:

$$
R(m)=y(m) \times \mathrm{W}_{\mathrm{RB}} \times \log _{2}(1+\mathrm{SNR}(m))
$$

where $y(m)$ is the share of resources allocated to user $m$. It is expressed in terms of RBs such as, $y(m)=\frac{n_{R B}(m)}{n_{R B}^{T o t a l}}$, where $n_{R B}(m)$ and $n_{R B}^{\text {Total }}$ are the number of allocated RBs to user $m$ and the maximum number of RBs available to the $\mathrm{BS}$, respectively. $\mathrm{W}_{\mathrm{RB}}$ is the bandwidth of one RB.

\section{BATTERY MODEL}

Compared with traditional used batteries, i.e., lead acid or nickel cadmium, lithium batteries technologies feature high energy density, high power density, large service life and environmental friendliness [8]. This new technology provides interesting efficiency improvements from $10 \%$ and $20 \%$ compared with other batteries. However, a typical used battery generates expensive investment cost due to its finite life span. This operational limitation is a result of irreversible degradations from chemical and physical changes, affecting its electrical performance, and hence, degrading its efficiency. In the 
following, we describe the constraints imposed on the battery that prevent its fast capacity degradation.

A typical battery is described by the following metrics:

1) SoC (State of Charge): It is the ratio of the remaining charge of the battery and the total charge while the battery is fully charged at the same specific standard condition [8].

3) DoD (Depth of Discharge): It is used to describe how deeply the battery is discharged. It is defined in percent. For instance, if the battery has delivered $70 \%$ of its energy, we say that the DoD of the battery is $70 \%$.

2) SoH (State of Health): it is a figure of merit that represents the condition of a battery compared to its ideal conditions [8]. We can define this metric as the ratio of the current capacity to the rated capacity:

$$
\operatorname{SoH}(t)=\frac{C_{r e f}(t)}{C_{N}}
$$

The degradation of the battery reference capacity is caused by two aging processes: during operation (i.e cycling), denoted by cycle aging and at rest, denoted by calendar aging [9]. Calculating the battery capacity degradation is not the focus of this work. However, we will use the constraints imposed on the battery to prevent such degradation.

1) Cycle aging: It is the result of the energy exchange with battery. It depends on the battery SoC variations [10]. Fig. 3 illustrates the ideal operating range of $\mathrm{SoC}$ that is recommended for lithium batteries [11]. As a result, the lithium battery usage is restricted to a specific range of:

$$
B_{L(t-1)+s}=[20 \%, 90 \%], \forall t=1, \ldots, T, \forall s=1, \ldots, L .
$$

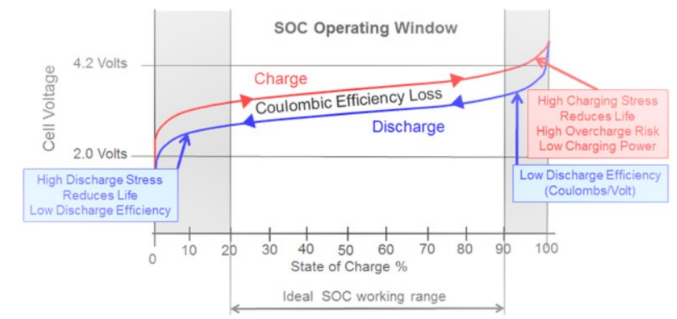

Fig. 3: Recommendation for the operating range of SoC for lithium ion battery [11].

2) Calendar aging: The battery calendar life is the time elapsed until the battery becomes unusable. It is influenced by the battery temperature and time [7]. Thus, in order to operate the lithium battery in a safe operating area restricted to temperature, high (dis)charge currents should be avoided. Consequently, the following constraint must be respected:

$$
\begin{aligned}
& \left|B_{L(t-1)+s}-B_{L(t-1)+s-1}\right| \leq \alpha \times B_{L(t-1)+s-1}, \\
& \forall t=1, \ldots, T, \forall s=1, \ldots, L, \backslash\{(t=1) \wedge(s=1)\} .
\end{aligned}
$$

where $\alpha$ takes a value between 0 and 1 .
In addition to the capacity degradation, the battery witnesses energy losses while (dis)charging. Each time the battery is (dis)charged with $\mathrm{E}$ units of energy, only $\eta \cdot E$ is used/stored, where $0 \leq \eta \leq 1$ represents the storage inefficiency.

\section{HyBRID ENERGy MANAGEMENT MODEL}

\section{A. Energy Consumption Model}

In order to calculate the power consumption of the BS, we use the power model provided by EARTH [12]. Accordingly, the power consumption of a BS consists of a static part $P_{0}$ and a traffic dependent part $P^{\mathrm{Tx}}$. Moreover, the power demand depends on the number of active RBs. Because of the nearly linear relationship that exists between the RF power and the BS power consumption, the power consumed by a BS at time slot $i$ can be expressed as follows:

$$
\begin{aligned}
& p(i)= \\
& \begin{cases}N_{\mathrm{Tx}}\left(P_{0}+y(i) \times \Delta_{p} \times P^{\mathrm{Tx}}(i)\right), & 0<P^{\mathrm{Tx}} \leq P_{\text {max }}, \\
N_{\mathrm{Tx}} \times P_{\text {sleep }}, & P^{\mathrm{Tx}}=0 .\end{cases}
\end{aligned}
$$

where $N_{\mathrm{Tx}}$ is the number of transceivers. $\Delta_{p}$ and $P_{\text {sleep }}$ are the load dependent parameter and the power consumption of the BS in sleep mode, respectively. $y(i)$ is the number of active RBs at time slot $i$.

\section{B. Energy Storage}

As shown in Fig. 1, the battery can store the harvested energy from the local PV system and the on-grid energy that is purchased from the smart grid. At each time slot, the local EMU decides how much energy to store from the RE source, and how much to buy from the grid, by jointly considering the BS energy demand, the hybrid energy storage, the price of electricity and the battery constraints imposed on the battery to prevent fast capacity degradation.

Denote the storage level of the battery at the beginning of slot $s$ of time $t$ as $B_{L(t-1)+s} \geq 0$, the amount of the grid energy purchased and stored in the battery as $p_{L(t-1)+s}^{g, b} \geq 0$, and the amount of power drawn from the battery as $p_{L(t-1)+s}^{b} \geq 0$. To make sure that the BS uses only the energy that is available before the beginning of each time slot, we need the following energy causality constraints:

$$
\begin{aligned}
& B_{L(t-1)+s}=B_{0}+\sum_{i=1}^{t} \sum_{j=1}^{s-1}\left(\eta\left(E_{L(i-1)+j}^{\mathrm{b}}+p_{L(i-1)+j}^{\mathrm{g}, \mathrm{b}}\right)\right. \\
& \left.-p_{L(i-1)+j}^{\mathrm{b}}-w_{L(i-1)+j}\right) \geq 0, \\
& \forall t=1, \ldots, T, \forall s=1, \ldots, L .
\end{aligned}
$$

The term $w$ introduced refers to the amount of RE lost at the end of the observed slot. This is due to the limited battery capacity, $B_{\max }$. Consequently, the battery has to discard the excess harvested energy $(w)$, to satisfy the following battery capacity constraints:

$$
B_{L(t-1)+s} \leq B_{\max }, \forall t=1, \ldots, T, \forall s=1, \ldots, L .
$$




\section{JOINT POWER \\ AND RESOURCE AlLOCATION: OFFLINE APPROACH}

Denote by $a(t)$ the real-time price of electricity at each time step $t$. We aim at minimizing the on-grid energy consumption of the energy harvesting BS taking into account the battery degradation model. This is achieved by jointly allocating the power between the power grid, the RE source, the battery, and the resources.

In the following, we formulate the offline power and resource allocation problem for the hybrid wireless system. The first set of optimization variables are the vector of power allocation $\mathbf{p}=\left(\mathbf{p}_{\mathbf{t}}\right)_{t=1, \ldots, T} \in \mathbb{R}_{+}^{T}$ used for the BS, and the matrix of resource allocation $\mathbf{y}=\left(\mathbf{y}_{\mathbf{t}, \mathbf{u}}\right)_{t=1 \ldots, T, u \in \mathcal{U}} \in \mathbb{R}_{+}^{T \times K}$ used for the served user. In addition, we optimize the following four power usage vectors for the BS: (1) the grid-BS power vector $\mathbf{p}^{\mathbf{g}, \mathbf{B S}}=\left(\mathbf{p}_{\mathbf{t}}^{\mathbf{g}, \mathbf{B S}}\right)_{t=1, \ldots, T} \in \mathbb{R}_{+}^{T}$ used from the power grid to power the $\mathrm{BS}$, (2) the batteryBS power vector $\mathbf{p}^{\mathbf{b}}=\left(\mathbf{p}_{\mathbf{t}}^{\mathbf{b}}\right)_{t=1, \ldots, T} \in \mathbb{R}_{+}^{T}$ used to power the BS from the battery, (3) the grid-battery power vector $\mathbf{p}^{\mathbf{g}, \mathbf{b}}=\left(\mathbf{p}_{\mathbf{t}}^{\mathbf{g}, \mathbf{b}}\right)_{t=1, \ldots T} \in \mathbb{R}_{+}^{T}$ bought from the power grid and stored in the battery for future use, and (4) the renewable power wastage vector $\mathbf{w}=\left(\mathbf{w}_{\mathbf{t}}\right)_{t=1, \ldots, T} \in \mathbb{R}_{+}^{T}$ that the BS will not be able to use or store. Finally, we optimize the use of RE vectors $\mathbf{E}^{B S}$ and $\mathbf{E}^{b}=\left(\mathbf{E}^{\mathbf{B S}}, \mathbf{E}^{\mathbf{b}}\right)_{t=1, \ldots, T} \in \mathbb{R}_{+}^{T}$.

$$
\begin{aligned}
& \min _{\substack{\mathbf{p}, \mathbf{y}, \mathbf{p}^{\mathbf{g}, \mathbf{B S}}, \mathbf{p}^{\mathbf{b}}, \mathbf{p}^{\mathbf{g}, \mathbf{b}}, \mathbf{E}^{\mathbf{B S}}, \mathbf{E}^{\mathbf{b}}\\
}} \sum_{t=1}^{T} \sum_{s=1}^{L} a_{t}\left(p_{L(t-1)+s}+p_{L(t-1)+s}^{g, b}\right. \\
& \left.-\eta p_{L(t-1)+s}^{b}-E_{L(t-1)+s}^{\mathrm{BS}}\right)
\end{aligned}
$$

Given that $p_{i}^{g, B S}=p_{i}-\eta \cdot p_{i}^{b}-E_{i}^{\mathrm{BS}}$, and from Eq.(2), we can rewrite (11) as follows:

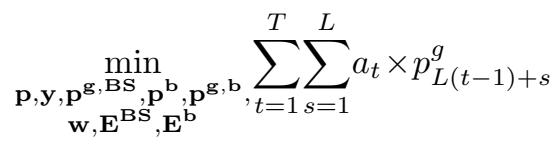

subject to:

$y_{L(t-1)+s, m} \times \mathrm{W}_{R B} \times \log _{2}\left(1+\mathrm{SNR}_{L(t-1)+s, m}\right) \geq R^{r e q}$, $\forall t=1, \ldots, T, \forall s=1, \ldots, L, \forall m \in \mathcal{U}$,

$\mathrm{E}_{L(t-1)+s}=E_{L(t-1)+s}^{\mathrm{b}}+E_{L(t-1)+s}^{\mathrm{BS}}+w_{L(t-1)+s}$,

$\forall t=1, \ldots, T, \forall s=1, \ldots, L$,

$p_{L(t-1)+s}^{\mathrm{g}}=p_{L(t-1)+s}^{\mathrm{g}, \mathrm{b}}+p_{L(t-1)+s}^{\mathrm{g}, \mathrm{BS}}$,

$\forall t=1, \ldots, T, \forall s=1, \ldots, L$,

$p_{L(t-1)+s}=p_{L(t-1)+s}^{g, B S}+\eta \cdot p_{L(t-1)+s}^{b}+\mathrm{RE}_{L(t-1)+s}^{B S}$,

$\forall t=1, \ldots, T, \forall s=1, \ldots, L$,

$\sum_{m \in \mathcal{U}} y_{L(t-1)+s, m} \leq 1, \forall t=1, \ldots T, \forall s=1, \ldots, L$,

$\mathbf{p} \geq 0, \mathbf{y} \geq 0, \mathbf{p}^{\mathbf{g}} \geq 0, \mathbf{p}^{\mathbf{b}} \geq 0, \mathbf{p}^{\mathbf{g}, \mathbf{B S}} \geq 0, \mathbf{p}^{\mathbf{g}, \mathbf{b}} \geq 0$,

$\mathbf{w} \geq 0, \mathbf{E}^{\mathbf{B S}} \geq 0, \mathbf{E}^{\mathbf{b}} \geq 0$,

Other Constraints: (6),(7),(9),(10).
The first set of constraints (13) guarantee a minimum rate requirement for the users in order to satisfy a certain QoS level. Next, we have the harvested energy usage, grid power usage and BS power equality constraints in (14), (15) and (16), respectively. Then, the resource allocation constraints are given in (17). Furthermore, the energy storage constraints are summarized in (9) and (10). the causal energy constraints (9) ensure that the total RE used up until the $i$-th time slot will not exceed the available amount that was harvested and stored in the previous $(i-1)$-th time slots. On the other hand, (10) is the battery capacity constraint. Consequently, in case of battery overflow, the excess energy must be discharged through the auxiliary variable w. Moreover, (6) and (7) describe the battery usage constraints. Finally, we have the non-negative vectors constraints in (18).

The offline optimization problem described above is a convex linear program. Hence, it can be solved efficiently using an optimization solver such as MATLAB using the dual-simplex algorithm. However, solving it requires the knowledge of all system variables in advance. In the next section, we will develop an online algorithm that achieves near-optimal solution without the requirement of future knowledge.

\section{BATTERY}

\section{Aging And Price-AwAre (BAPA) Algorithm}

In this section, we provide an online solution based on the design philosophy of the offline algorithm. We propose BAPA, a new algorithm that does not require the knowledge of RE generation. We decompose the problem into three subproblems: Resource sharing problem, grid energy purchase problem and power allocation problem. Then, we summarize our algorithm in Algorithm 1.

\section{A. Resource Sharing Problem (Max-Min Fairness Algorithm)}

In order to share the BS radio resources among the users it is serving to satisfy their QoS requirements, we use the Max-Min fairness algorithm detailed in [13], [14]. The algorithm starts by computing the share of resources needed for each user. Then, it divides the resources evenly among them. If a user gets more resources than his requirement, additional resources will be divided among other users that have not yet achieved their requirements. If all users are satisfied, excess radio resources will be turned off to save energy. In our model, we consider that the BS has a fixed number of resources expressed in terms of RBs.

\section{B. Grid Energy Purchase Problem}

According to Eq. (8), the minimum amount of power required to power the $\mathrm{BS}$ during the observation time $T$ is: 


$$
\begin{aligned}
& P_{L(t-1)+s}^{\min }=\sum_{i=t}^{T} \sum_{j=s}^{L} p_{L(i-1)+j}^{\min } \\
& =\sum_{i=t}^{T} \sum_{j=s}^{L} N_{T x}\left(P_{0}+\frac{1}{n_{R B}^{T o t a l}} \times \Delta_{p} \times P_{L(i-1)+j}^{\mathrm{Tx}}\right)
\end{aligned}
$$

Eq. (19) gives the minimum power required for the $\mathrm{BS}$ to be in operational mode, i.e., by serving only one $\mathrm{RB}$, from time $t$ until the end of the day.

Proposition 1 (sub-optimal on-grid energy purchase): The amount of purchased energy from the grid should satisfy the following conditions:

1) Condition \#1: Low current on-grid electricity price. $a(t) \leq \min (a(t+1), \ldots, a(T))$.

2) Condition \#2: Low future renewable energy. $\sum_{i=t}^{T} \sum_{j=s}^{L} E_{L(i-1)+j} \leq P_{L(t-1)+s}^{\min }$.

If the above conditions are satisfied:

$$
\begin{aligned}
& p_{L(t-1)+s}^{g, b}=\min \left(\left(B_{\max }-B_{L(t-1)+s}\right) \times \alpha,\right. \\
& \left.\left(P_{L(t-1)+s}^{\min }-\sum_{i=t}^{T} \sum_{j=s}^{L} E_{L(i-1)+j}\right) \times \alpha\right) .
\end{aligned}
$$

where $\alpha$ is the current restriction coefficient that limits the (dis)charging rate of the battery to keep it in a safe operational area restricted to temperature. It will not allow the battery to be (dis)charged more than $\alpha \%$ of its current capacity.

Proposition 1 decides when to purchase grid energy, and how much to store in the battery. Condition \# 1 allows buying grid energy only when the price is at its lowest compared to the future price. This policy is in accord with the daily grid energy price that is low at the beginning of the day and assumes future knowledge of the electricity price [15]. However, condition \# 2 requires the exact amount of RE that will be harvested in the future. Since this information is not known, we will predict this energy using weather forecast programs, such as [16]. Thus, we can rewrite condition \# 2 as follows:

$$
G_{L(t-1)+s} \leq P_{L(t-1)+s}^{\min }
$$

where $G_{t}$ is the green energy budget predicted from time $t$ until the end of the observation time.

\section{Power Allocation Problem}

The power allocation problem is divided into two parts. The first part (Line 10) finds the allowed amount of RE that can be stored in the battery $\left(E^{\mathrm{b}}\right)$, while respecting the battery constraints in (6) and (7). The second part (Lines 11-18) decides whether to use the battery to power the BS or save its energy for future use (i.e., when there is enough battery).
Algorithm 1: BAPA algorithm

1: Initialization: Set the battery specific range, its current restriction parameter $(\alpha)$ and its DoD.

2: Predict the green energy budget, $G(1)$, for the whole day.

3: for $t=1: T$ do

4: $\quad$ for $s=1: L$ do

$\backslash \backslash$ Subproblem 1: Resource Sharing Problem

5: Obtain $y_{L(t-1)+s}$ by solving Max-Min algorithm;

$\backslash$ Subproblem 2: Grid Energy Purchase

\section{Problem}

6: $\quad$ Find $p_{L(t-1)+s}$ from Eq. (8);

7: $\quad$ if conditions 1 and 2 in proposition 1 are satisfied then

8: $\quad$ Find $p_{L(t-1)+s}^{\mathrm{g}, \mathrm{b}}$ from Eq. (20);

9: $\quad$ end if

$\backslash \backslash$ Subproblem 3: Power Allocation Problem

10: $\quad$ Find $E_{L(t-1)+s}^{\mathrm{b}}$ and $E_{L(t-1)+s}^{\mathrm{BS}}$

from Eq. (1), $p_{L(t-1)+s}$ calculated in line

6 and the battery constraints (6) and (7);

11: $\quad$ if $G_{L(t-1)+s} \geq P_{L(t-1)+s}^{\min }$ then (use battery)

12: $\quad$ Find $p_{L(t-1)+s}^{\mathrm{b}}$, such that $p_{L(t-1)+s}=$ $\eta \cdot p_{L(t-1)+s}^{\mathrm{b}}+E_{L(t-1)+s}^{\mathrm{b}}$, while satisfying (6), (7) and (9);

13: $\quad$ Update $B_{L(t-1)+s}=B_{L(t-1)+s}+$ $\eta \cdot E_{L(t-1)+s}^{\mathrm{b}}-p_{L(t-1)+s}^{\mathrm{b}}$;

14: $\quad$ Find $p_{L(t-1)+s}^{\mathrm{g}, \mathrm{BS}}=p_{L(t-1)+s}-$ $E_{L(t-1)+s}^{\mathrm{BS}}-p_{L(t-1)+s}^{\mathrm{b}}$;

15: $\quad$ else (store in the battery)

16: $\quad$ Find $B_{L(t-1)+s}=B_{L(t-1)+s}+$ $\eta \cdot E_{L(t-1)+s}^{\mathrm{b}}$;

17: $\quad$ Find $p^{\mathrm{g}, \mathrm{BS}}=p_{L(t-1)+s}-E_{L(t-1)+s}^{\mathrm{BS}}$;

18: $\quad$ end if

19: end for

20: end for

\section{Simulation AND ReSUlts}

We consider a macro BS covering a radius of 500 $m$ equipped with a RE source and an energy storage (i.e., battery). The users are uniformly distributed in the simulation area with a maximum of 20 users requesting simultaneously data from the designated BS. We use a lithium type battery with a DoD of $70 \%$. Furthermore, we restrict the (dis)charging battery rate $\alpha=0.3$. In other words, during one hour, the battery cannot be (dis)charged more than $30 \%$ of its current capacity. In the following, we compare BAPA with two benchmark algorithms: the optimal solution provided in Section $\mathrm{V}$ and an online algorithm called SPAEMA that we introduced in [4]. The latter decides when to store RE and when to use it based on the SoC of the battery and the current price of electricity. Table I details the assumptions and parameters used in our simulation.

In Fig. 4, we plot the normalized grid energy consumption of the BS for different daily average 
TABLE I: Parameters' values and assumptions.

\begin{tabular}{|c|c|}
\hline Parameters & Values \\
\hline BS area radius R & $500 \mathrm{~m}$ \\
\hline Number of sectors & 3 \\
\hline Bandwidth & $10 \mathrm{MHz}, \mathrm{FDD}$ \\
\hline Maximum transmitted power & $43 \mathrm{dBm}$ \\
\hline RB & 50 \\
\hline $\mathrm{W}_{\mathrm{RB}}$ & $180 \mathrm{KHz}$ \\
\hline Max. number of users K & 20 \\
\hline User min. required rate & $4 \mathrm{Mb} / \mathrm{s}$ \\
\hline Noise power & $-174 \mathrm{dBm} / \mathrm{Hz}$ \\
\hline$P_{0}$ & $118.7 \mathrm{~W}$ \\
\hline$\Delta_{p}$ & 5.32 \\
\hline Number & 10 \\
\hline of slots per hour period $\mathrm{L}$ & \\
\hline Battery current restriction $\alpha$ & 0.3 \\
\hline Battery energy efficiency $\eta$ & 0.96 \\
\hline
\end{tabular}

$\mathrm{RE}$ generation rates $\left(\mathrm{RE}_{\text {Total }}\right)$, where $\mathrm{E}_{\text {Total }}$ is the average daily energy demand of the BS. The grid energy consumption is normalized with the BS energy demand at full load. Furthermore, we size the battery capacity in proportion to the PV cell installed. In other words, the bigger the solar panel is, the larger the battery capacity becomes. In the following, we consider three PV pane sizes, each with different daily average RE generation rate. First, BAPA consumes excess grid energy at the beginning of the day, as shown at $t=1,3,4,5$. During these periods, our algorithm buys energy from the grid and stores it in the battery for future use. By inspecting the grid energy purchase policy in proposition 1, we observe that the grid energy is at its lowest during these periods. hence condition \#1 is satisfied. Regarding condition \#2, we equip the BS with PV cells that harvest energy less than the demand required by the BS during the day. Thus, at the beginning the day, condition \#2 is always satisfied. Second, we observe that the bigger the PV cel] is (bigger battery capacity, resp.), the more the system is able to store grid energy during the first hours of the day. This is because BAPA restricts the charging rate of the battery based on its current capacity, in order to preserve its $\mathrm{SoH}$. As a result, small batteries will not be able to store enough grid power for future use.

In Fig. 5, we compare our algorithm (BAPA) with the optimal solution and SPAEMA. We observe that the performance of our algorithm is close to the optimal energy and resource allocation problem (up to 99\%), whereas SPAEMA diverges away from optimality. The reason is twofold: First, BAPA allows the possibility to store grid energy when the price is low and to use it later at high energy prices. Second, SPAEMA is a threshold based algorithm that depends on fixed battery SoC and current energy price thresholds. Hence, increasing the battery capacity for instance, forces SPAEMA to store more energy until the threshold of using RE is reached. BAPA, on the other hand, depends on the current price of electricity with respect to future prices, and on the difference between the available RE and required energy demand. This results in a more accurate RE utilization

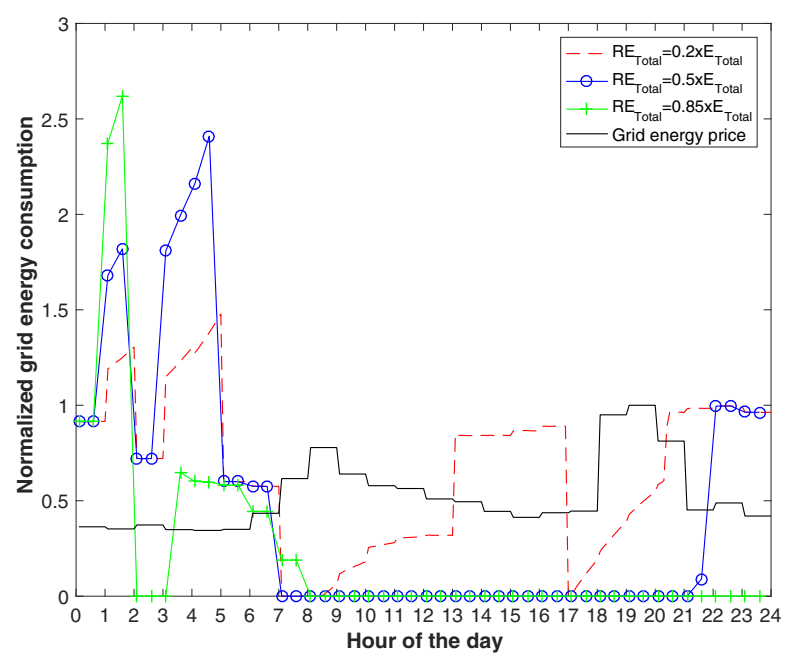

Fig. 4: Grid energy consumption for different PV cell sizes.

compared with SPAEMA.

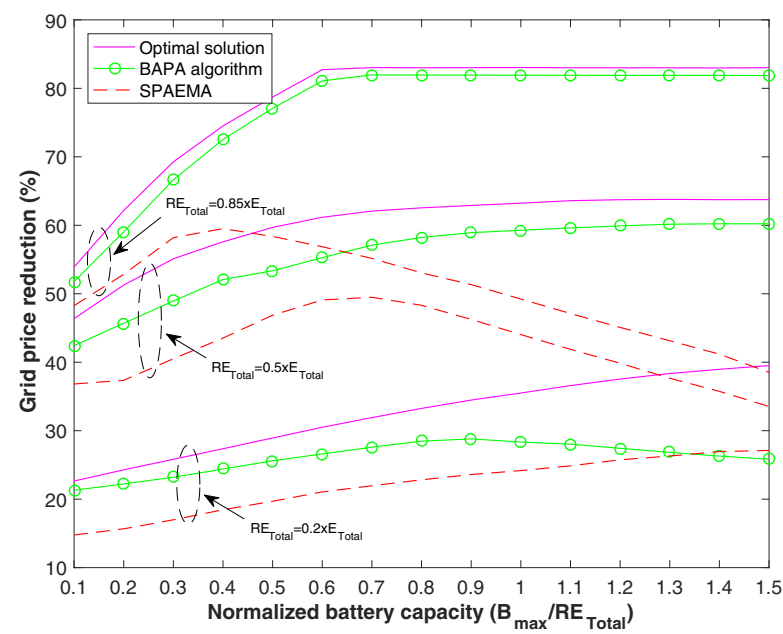

Fig. 5: Performance evaluation of BAPA algorithm with the optimal solution and a benchmark online algorithm.

We illustrate the waste of energy in Fig. 6. We consider the case of a solar panel harvesting $50 \%$ of the total BS energy demand. We observe that for high battery capacities, BAPA shows slightly higher energy losses than SPAEMA (4\% points). The reason is that BAPA algorithm stores energy from the grid. However, due to the battery inefficiency, some of this energy is lost, resulting in additional waste in energy compared with SPAEMA.

BAPA algorithm relies on the RE predicted throughout the day. As discussed in Section VI-B, this prediction is based on weather forecast programs, and thus is prone to errors. In Fig. 7, we show that a prediction error of $10 \%$ in the harvested RE degrades the performance of BAPA by less than $1 \%$ point. Whereas a prediction error of $30 \%$ decreases the grid price reduction to about $7 \%$ points. 


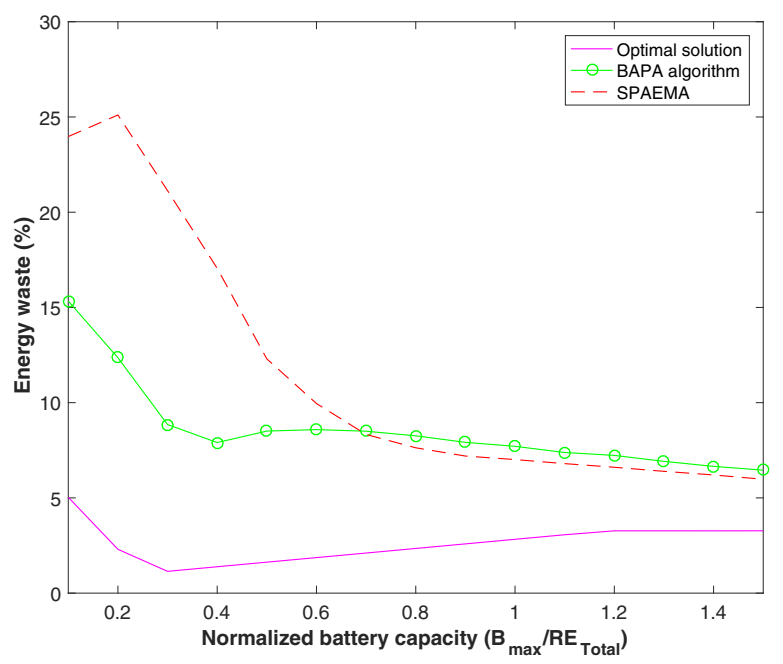

Fig. 6: Energy waste with solar panel that harvests $50 \%$ of the total BS energy demand.

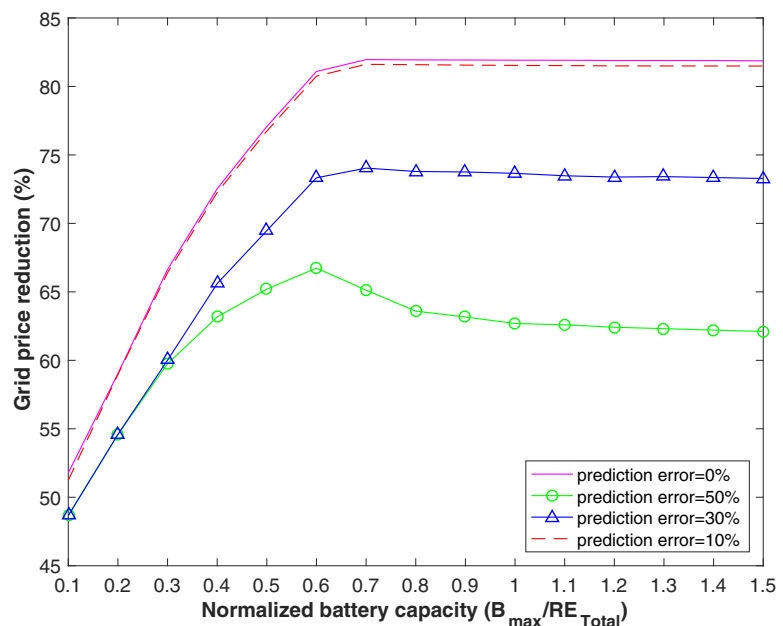

Fig. 7: On-grid price reduction with different harvested energy prediction errors.

\section{CONCLUSION}

In this paper, we have addressed the problem of grid energy consumption in hybrid BS cellular network equipped with RE. In contrast to most studies that focus only on reducing the opex cost of the operator by bringing down the grid energy consumption of the network, our work takes into account the battery degradation model that represents a significant cost to the operator. We proposed BAPA, an energy management algorithm that brings down the grid energy consumption of the operator, while preserving the life of the battery. Our results show that BAPA can reach up to $99 \%$ grid energy savings compared to the optimal solution and outperforming a benchmark algorithm.

This work opens a new axis for future research. Energy storages represent expensive capex and opex costs for cellular network operators. Thus, careful utilization of these elements is essential and should not be ignored. For future work, we plan to perform a detailed study on the long term evolution of the system performance under the battery aging model. This would provide interesting recommendations to future green cellular networks.

\section{ACKNOWLEDGMENTS}

The authors would like to acknowledge the support of the French Ministry of Industry for supporting this work under SooGREEN project.

\section{REFERENCES}

[1] L. Suarez, L. Nuaymi, and J.-M. Bonnin, "An overview and classification of research approaches in green wireless networks," EURASIP Journal on Wireless Communications and Networking, vol. 2012, p. 142, Apr 2012.

[2] E. Gelenbe and Y. Caseau, "The impact of information technology on energy consumption and carbon emissions," vol. 2015, pp. 1-15, June 2015.

[3] H. A. H. Hassan, L. Nuaymi, and A. Pelov, "Renewable energy in cellular networks: A survey," in 2013 IEEE Online Conference on Green Communications (OnlineGreenComm), pp. 1-7, Oct 2013.

[4] H. A. H. Hassan, L. Nuaymi, and A. Pelov, "Classification of renewable energy scenarios and objectives for cellular networks," in 2013 IEEE 24th Annual International Symposium on Personal, Indoor, and Mobile Radio Communications (PIMRC), pp. 2967-2972, Sept 2013.

[5] H. A. H. Hassan, A. Pelov, and L. Nuaymi, "Cost-efficient radio resource allocation in hybrid energy cellular networks," in 2014 IEEE Global Communications Conference, pp. 2472-2478, Dec 2014.

[6] R. Ramamonjison and V. K. Bhargava, "Energy allocation and cooperation for energy-efficient wireless two-tier networks," IEEE Transactions on Wireless Communications, vol. 15, pp. 6434-6448, Sept 2016.

[7] M. Mendil, A. De Domenico, V. Heiries, R. Caire, and N. Hadjsaid, "Battery aging-aware energy management of green small cells powered by the smart grid," EURASIP Journal on Wireless Communications and Networking, vol. 2017, p. 127, Jul 2017.

[8] L. Lu, X. Han, J. Li, J. Hua, and M. Ouyang, "A review on the key issues for lithium-ion battery management in electric vehicles," Journal of Power Sources, vol. 226, pp. 272 - 288, 2013.

[9] M. Broussely, P. Biensan, F. Bonhomme, P. Blanchard, S. Herreyre, K. Nechev, and R. Staniewicz, "Main aging mechanisms in li ion batteries," Journal of Power Sources, vol. 146 , no. 1 , pp. $90-96,2005$. Selected papers pressented at the 12th International Meeting on Lithium Batteries.

[10] Y. Riffonneau, S. Bacha, F. Barruel, and S. Ploix, "Optimal power flow management for grid connected pv systems with batteries," IEEE Transactions on Sustainable Energy, vol. 2, pp. 309-320, Jul 2011.

[11] Lithium battery failures. [Online]. Available: http://mpoweruk.com/lithium_failures.htm\#soc.

[12] G. Auer, V. Giannini, C. Desset, I. Godor, P. Skillermark, M. Olsson, M. A. Imran, D. Sabella, M. J. Gonzalez, O. Blume, and A. Fehske, "How much energy is needed to run a wireless network?," IEEE Wireless Communications, vol. 18, pp. 40-49, Oct 2011.

[13] M. Ghorbanzadeh, A. Abdelhadi, and C. Clancy, Utility Functions and Radio Resource Allocation, pp. 21-36. Cham: Springer International Publishing, 2017.

[14] A. El-Amine, H. A. H. Hassan, and L. Nuaymi, "Analysis of energy and cost savings in hybrid base stations power configurations," in 2018 IEEE 87th Vehicular Technology Conference (VTC Spring), pp. 1-7, June 2018.

[15] Epex, european power exchange spot information. [Online]. Available: http://www.epexspot.com.

[16] PVWatts calculator. [Online]. Available: http://www.pvwatts. nrel.gov. 